九州大学学術情報リポジトリ

Kyushu University Institutional Repository

\title{
ON OPTIMALITY CONDITIONS FOR TRILEVEL DYNAMIC OPTIMIZATION PROBLEMS
}

Shao, $\mathrm{Yi}$-Hang

Department of Mathematics, Saitama University

https://doi.org/10.5109/13485

出版情報: Bulletin of informatics and cybernetics. 31 (2), pp.117-136, 1999-12. Research Association of Statistical Sciences

バージョン :

権利関係 : 


\title{
ON OPTIMALITY CONDITIONS FOR TRILEVEL DYNAMIC OPTIMIZATION PROBLEMS
}

By

\section{Yi-Hang SHAO*}

\begin{abstract}
In this paper, we introduce trilevel dynamic optimization problems. Reformulating the trilevel dynamic problem as a single-level optimal control problem with state-control functional constraints, we derive the necessary optimality conditions. We ajso show that the necessary conditions are sufficient for optimality in a 'convex' case.
\end{abstract}

Key Words and Phrases: trilevel dynamic optimizaion problems, nonsmooth analysis, necessary conditions, sufficient conditions, constraint qualifications.

\section{Introduction}

In this paper, we consider the following trilevel dynamic optimization problem $(T D O P)$. In this problem, there are three players $A, B$ and $C$ whose controls are $u$, $v$ and $w$, respectively. The players $A, B$ and $C$ minimize their cost functionals on the following manner:

For each $u$ chosen by the player $A$, the player $B$ selects a control $v=v[u] \in \mathcal{V}[u]$, where $\mathcal{V}[u]$ is the set of optimal controls $v$ of the following optimal control problem,

$$
\begin{aligned}
P_{B}[u]: \quad \text { Minimize: } & \int_{0}^{1} G_{1}(t, y(t), u(t), v(t)) d t+g_{1}(y(1)) \\
\text { subject to : } & \dot{y}(t)=\varphi(t, y(t), u(t), v(t)) \quad \text { a.e. } \\
& y(0) \in C_{2} \\
& v(t) \in U_{2}(t) \quad \text { a.e.. }
\end{aligned}
$$

Next, the player $C$ chooses a control $w=w[u, v] \in \mathcal{W}[u, v](v=v[u])$, where $\mathcal{W}[u, v]$ is the set of optimal controls $w$ of the following problem.

$$
\begin{aligned}
P_{C}[u, v]: \text { Minimize: } & \int_{0}^{1} G_{2}(t, z(t), u(t), v(t), w(t)\} d t+g_{2}(z(1)) \\
\text { subject to: }: & \dot{z}(t)=\psi(t, z(t), u(t), v(t), w(t)) \quad \text { a.e. } \\
& z(0) \in C_{3} \\
& w(t) \in U_{3}(t) \quad \text { a.e.. }
\end{aligned}
$$

* Department of Mathematics, Saitama University, Japan. 
Among all controls $(u, v, w)=(u, v[u], w[u, v[u]])$, the player $A$ selects a control optimizing the following optimal control problem,

$$
\begin{aligned}
P_{A}: \text { Minimize: } & \int_{0}^{1} F(t, x(t), u(t), v(t), u(t)) d t+f(x(1)) \\
\text { subject to: } \quad & \dot{x}(t)=\phi(t, x(t), u(t), v(t), u(t)) \\
& x(0) \in C_{1} \\
& u(t) \in C_{1}(t) \quad \text { a.e. } \\
& v \in \mathcal{V}[u] \\
& w \in \mathcal{W}[u, v] .
\end{aligned}
$$

In this trilevel dynamic optimization problem $(T D O P)$,

$$
\left.\begin{array}{l}
(x(\cdot), y(\cdot), z(\cdot)) \in A C\left([0,1], R^{m_{1}} \times R^{m_{2}} \times R^{m_{3}}\right) \text { is the state, } \\
(u(\cdot), v(\cdot), w(\cdot)) \in L^{1}\left([0,1], R^{n_{1}} \times R^{n_{2}} \times R^{n_{3}}\right) \text { is the control, } \\
F:[0,1] \times R^{m_{1}} \times R^{n_{1}} \times R^{n_{2}} \times R^{n_{3}} \rightarrow R, \\
f: R^{m_{1}} \rightarrow R, \\
G_{1}:[0,1] \times R^{m_{2}} \times R^{n_{1}} \times R^{n_{2}} \rightarrow R, \\
g_{1}: R^{m_{2}} \rightarrow R, \\
G_{2}:[0,1] \times R^{m_{3}} \times R^{n_{1}} \times R^{n_{2}} \times R^{n_{3}} \rightarrow R, \\
g_{2}: R^{m_{3}} \rightarrow R, \\
\phi:[0,1] \times R^{m_{1}} \times R^{n_{1}} \times R^{n_{2}} \times R^{n_{3}} \rightarrow R^{m_{1}}, \\
\\
\varphi:[0,1] \times R^{m_{2}} \times R^{n_{1}} \times R^{n_{2}} \rightarrow R^{m_{2}}, \\
\psi:[0,1] \times R^{m_{3}} \times R^{m_{1}} \times R^{n_{2}} \times R^{n_{3}} \rightarrow R^{m_{3}}
\end{array}\right\} \text { are given functions, }
$$

where $A C\left([0,1], R^{m_{1}} \times R^{m_{2}} \times R^{m_{3}}\right)$ denotes the space of absolutely continuous functions on $[0,1]$ with value in $R^{m_{1}} \times R^{m_{2}} \times R^{m_{3}}$.

A control $(u, v, w)$ of $(T D O P)$ corresponding to the state $(x, y, z)$ is called admissible iff $(x, u, v, w)$ satisfies the differential equation $\dot{x}=\phi(t, x, u, v, w)$ a.e. with initial condition $x(0) \in C_{1}$ and control constraint $u(t) \in U_{1}(t)$ a.e., $(y, v)$ and $(z, w)$ are optimal solution for $P_{B}[u]$ and $P_{C}[u, v]$ respectively. An admissible control $\left(u_{*}, v_{*}, w_{*}\right)$ corresponding to state $\left(x_{*}, y_{*}, z_{*}\right)$ is called optimal for $(T D O P)$ iff $\left(x_{*}, u_{*}, v_{*}, w_{*}\right)$ minimizes the value of cost functional of $P_{A}$ over all admissible controls corresponding to the states of $(T D O P)$.

This trilevel dynamic optimization problem can be applied in various areas. For instance, in economics, the controls $u$ are government's monetary or fiscal policies: the controls $v$ are decisions of consumers which respond to the policies $u$; the controls $w$ are decisions of firms which depend on the policies of government $u$ and the consumer's decisions $v$.

Many papers have been devoted to bilevel programming problems (static optimization). Yezza (1996) studied necessary optimality conditions for multilevel programming problem. For the bilevel dynamic optimization problems, the recent results are given by Ye $(1995,1997)$. In these papers, under some assumptions, Ye reduced the bilevel 
dynamic optimization problems to single-level optimal control problems without statecontrol constraints. Then, Ye derived the necessary optimality conditions.

To our knowledge. there is no paper dealing with optimality conditions for general trilevel optimal control problems. The main purpose of our paper is to discuss the necessary optimality conditions for the above trilevel dynamic optimization problem in general case. This problem can not be reduced to a single-level optimal control problem which has no state-control constraints. Thus, we can not extend the results of necessary conditions in Ye $(1995,1997)$ to our $(T D O P)$. Noreover, we show that our necessary conditions are also sufficient for optimality under some convexity assumptions on the functions and sets in $(D)$. Using the results given below, we can also derive the optimality conditions for another type trilevel optimization problems (see Remark (i) below). These conclusions can be generalized easily to $k$-level dynamic optimization problems without other additional hypothesis.

Define the value function $V_{1}(u): L^{1}\left([0,1], R^{m_{1}}\right) \rightarrow R \cup\{+\infty\} \cup\{-\infty\}$ for $P_{B}[u]$ by:

$$
\begin{aligned}
V_{1}(u):= & \inf \left\{\int_{0}^{1} G_{1}(t, y(t), u(t), v(t)) d t+g_{1}(y(1)):\right. \\
& \left.\dot{y}(t)=\varphi(t, y(t), u(t), v(t)) \text { a.e., } y(0) \in C_{2}, v(t) \in U_{2}(t) \text { a.e. }\right\},
\end{aligned}
$$

the value function $V_{2}(u, v): L^{1}\left([0,1], R^{m_{1}}\right) \times L^{1}\left([0,1], R^{m_{2}}\right) \rightarrow R \cup\{+\infty\} \cup\{-\infty\}$ for $P_{C}[u, v]$ by:

$$
\begin{aligned}
V_{2}(u, v):= & \inf \left\{\int_{0}^{1} G_{2}(t, z(t), u(t), v(t), w(t)) d t+g_{2}(z(1)):\right. \\
& \left.\dot{z}(t)=\dot{\psi}(t, z(t), u(t), v(t), w(t)) \text { a.e., } z(0) \in C_{3}, w(t) \in U_{3}(t) \text { a.e. }\right\} .
\end{aligned}
$$

Throughout this paper, by convention, we assume that the infimum over empty set is $+\infty$.

Then, the above problem $(T D O P)$ is obviously equivalent to the following singlelevel optimal control problem,

$$
\begin{aligned}
P: \quad \text { Minimize : } & \int_{0}^{1} F(t, x(t), u(t), v(t), w(t)) d t+f(x(1)) \\
\text { subject to : } & \dot{x}(t)=\phi(t, x(t), u(t), v(t), w(t)) \quad \text { a.e. } \\
& \dot{y}(t)=\varphi(t, y(t), u(t), v(t)) \quad \text { a.e. } \\
& \dot{z}(t)=\psi(t, z(t), u(t), v(t), w(t)) \quad \text { a.e. } \\
& (x(0), y(0), z(0)) \in C_{1} \times C_{2} \times C_{3} \\
& (u(t), v(t), w(t)) \in U_{1}(t) \times U_{2}(t) \times U_{3}(t) \quad \text { a.e. } \\
& \int_{0}^{1} G_{1}(t, y(t), u(t), v(t)) d t+g_{1}(y(1))-V_{1}(u) \leq 0 \\
& \int_{0}^{1} G_{2}(t, z(t), u(t), v(t), w(t)) d t+g_{2}(z(1))-V_{2}(u, v) \leq 0,
\end{aligned}
$$

which contains state-control constraints in which the value functions are nonsmooth in general. We shall study the optimality conditions for such optimal control problem in the next section. 


\section{Nonsmooth Optimal Control Problem}

In this section, we deal with the following problem:

$$
\begin{aligned}
(\text { NOCP }): \quad \text { Minimize }: & \int_{0}^{1} L_{0}(t, x(t), u(t)) d t+h_{0}(x(1)) \\
\text { subject to }: & \dot{x}(t)=\Phi(t, x(t), u(t)) \quad \text { a.e. } \\
& x(0) \in C \\
& u(t) \in U(t) \quad \text { a.e. } \\
& \bar{G}_{i}(x, u) \leq 0 \quad i=1, \cdots, k,
\end{aligned}
$$

in which

$$
\bar{G}_{i}(x, u):=\int_{0}^{1} L_{i}(t, x(t), u(t)) d t+h_{i}(x(1))-\Lambda_{i}(u) .
$$

Here, $x(\cdot) \in A C\left([0,1], R^{m}\right), u(\cdot) \in L^{1}\left([0,1], R^{n}\right), L_{i}:[0,1] \times R^{m} \times R^{n} \rightarrow R, h_{i}: R^{m} \rightarrow$ $R(i=0, \cdots, 1), \Lambda_{i}: L^{1} \rightarrow R \cup\{+\infty\} \cup\{-\infty\}(i=1, \cdots, k), \Phi:[0,1] \times R^{m} \times R^{n} \rightarrow R^{m}$, $C$ is a subset of $R^{m}$ and $U:[0,1] \rightarrow 2^{R^{n}}$.

We say that $(x, u) \in A C \times L^{1}$ is an admissible process for (NOCP) iff $L_{i}(\cdot, x(\cdot), u(\cdot))$ $(i=0, \cdots, k)$ are integrable and $(x, u)$ satisfies all constraints in (NOCP). An admissible process $\left(x_{*}, u_{*}\right)$ is called a local minimizer for $(N O C P)$ iff $\left(x_{*}, u_{*}\right)$ minimizes the cost over all admissible processes $(x, u)$ satisfying $\left\|x-x_{*}\right\|_{L^{*}}<\epsilon$ and $\left\|u-u_{k^{*}}\right\|_{L^{1}}<\epsilon$ for some $\epsilon>0$.

\subsection{Necessary conditions}

Letting $\left(x_{*}, u_{*}\right)$ be an admissible process for $(N O C P)$, we assume that $\{C, U(t)$, $\left.\Phi, L_{i}, h_{i}(i=0, \cdots, 1), \Lambda_{i}(i=1, \cdots, k)\right\}$ satisfies (A1)-(A6) below.

(A1): $C$ is a closed subset of $R^{m}$.

(A2): $U(\cdot)$ is a closed set-valued map, $\operatorname{Gr} U$ is $\mathcal{L} \times \mathcal{B}$ measurable. There exists $\rho(\cdot) \in L^{1}$, such that $|u| \leq \rho(t)$ for any $u \in U(t)$ a.e. $t \in[0,1]$, where $|u|$ is the Euclidean norm for $u \in R^{m}$.

(A3): $\Phi(t, x, u)$ is measurable in $t$, continuously differentiable in $(x, u)$. There exists $k(t)$ $\epsilon L^{\infty}$ and $\epsilon>0$ such that for almost all $t$

$$
\left|\Phi\left(t, x_{1}, u_{1}\right)-\Phi\left(t, x_{2}, u_{2}\right)\right| \leq k(t)\left(\left|x_{1}-x_{2}\right|+\left|u_{1}-u_{2}\right|\right)
$$

for $x_{1}, x_{2} \in x_{*}(t)+\epsilon B_{R^{m}}, u_{1}, u_{2} \in R^{n} . B_{R^{m}}$ is the closed unit ball of $R^{m}$.

(A4): $L_{0}, \cdots, L_{k}$ are measurable in $t$, and there exists $k_{L}(\cdot) \in L^{\infty}$ such that for any $i \in$ $\{0, \cdots, k\}$

$$
\left|L_{i}\left(t, x_{1}, u_{1}\right)-L_{i}\left(t, x_{2}, u_{2}\right)\right| \leq k_{L}(t)\left(\left|x_{1}-x_{2}\right|+\left|u_{1}-u_{2}\right|\right)
$$

for all $x_{1}, x_{2} \in x_{*}(t)+\epsilon B_{R^{m}}, u_{1}, u_{2} \in R^{n}$ a.e. $t \in[0,1]$.

(A5): There is $k_{h}>0$ such that for each $i \in\{0, \cdots, 1\}$;

$$
\left|h_{i}\left(x_{1}\right)-h_{i}\left(x_{2}\right)\right| \leq k_{h}\left|x_{1}-x_{2}\right|
$$


for any $x_{1}, x_{2} \in x_{*}(1)+\epsilon B_{R^{\prime \prime \prime}}$.

(A6): There exists $k_{\mathrm{I}}>0$ such that for any $i \in\{1, \cdots k\}$.

$$
\left|\Lambda_{i}\left(u_{1}\right)-\Lambda_{i}\left(u_{2}\right)\right| \leq k_{\mathrm{A}}\left\|u_{1}-u_{2}\right\|_{L^{1}}
$$

for any $u_{j}(\cdot)$ with $\left\|u_{j}-u_{w_{*}}\right\|_{L^{1}} \leq \epsilon, j=1,2$.

Now, we state our main theorem for necessary optimality conditions. For simplicity, we abbreviated the arguments $\left(t, x_{*}(t), u_{*}(t)\right)$ to $[t]$, for instance $\Phi[t]:=\Phi\left(t, x_{*}(t), u_{*}(t)\right)$.

ThEOREM 2.1. Let $\left(x_{*}(\cdot), u_{*}(\cdot)\right)$ be a local minimizer for (NOCP). Assume that (A1)-(A6) are satisfied. Then, there exist $\lambda_{i} \geq 0(i=0, \cdots, k)$ with $\sum_{i=0}^{k} \lambda_{i}=1$, $p(\cdot) \in A C\left([0,1], R^{m}\right)$ and $\zeta(\cdot) \in L^{\infty}\left([0,1], R^{n}\right)$ such that

$$
\begin{gathered}
(-\dot{p}(t), \zeta(t)) \in \nabla_{(x, u)}(p(t), \Phi[t]\rangle-\sum_{i=1}^{k} \lambda_{i} \partial_{(x, u)} L_{i}[t] \quad \text { a.e. } \\
\zeta=\zeta_{1}+\zeta_{2}, \quad-\zeta_{1} \in \sum_{i=1}^{k} \lambda_{i} \partial \Lambda_{i}\left(u_{*}(\cdot)\right), \quad \zeta_{2}(t) \in N_{L(t)}\left(u_{*}(t)\right) \text { a.e. } \\
p(0) \in N_{C}\left(x_{*}(0)\right), \quad-p(1) \in \sum_{i=1}^{k} \lambda_{i} \partial h_{i}\left(x_{*}(1)\right) \\
\lambda_{i}\left(\int_{0}^{1} L_{i}[t] d t+h_{i}\left(x_{*}(1)\right)-\Lambda_{i}\left(u_{*}\right)\right)=0(i=1, \cdots, k) .
\end{gathered}
$$

In particular, assume that there exists $\left(x_{1}, u_{1}\right) \in A C \times L^{1}$ such that the following constraint qualifications (2.5) and (2.6) hold,

$$
\begin{gathered}
\int_{0}^{1} L_{i}{ }^{\circ}\left(\left(x_{*}, u_{*}\right),\left(x_{1}, u_{1}\right)\right) d t+h_{i}{ }^{\circ}\left(x_{*}, x_{1}\right)+\left(-\Lambda_{i}\right)^{\circ}\left(u_{*}, u_{1}\right)<0 \quad \text { for } i \in I_{*} \\
\dot{x}_{1}=\Phi_{x}[t] x_{1}+\Phi_{u}[t] u_{1} \quad \text { a.e., } x_{1}(0) \in T_{C}\left(x_{*}(0)\right) \text { and } u_{1}(t) \in T_{U(t)}\left(u_{*}\right) \text { a.e., }
\end{gathered}
$$

where, $I_{*}:=\left\{i \in[1, \cdots, k]: \bar{G}_{i}[t]=0\right\}$. Then, we have $\lambda_{0}>0$.

We will give the proof of this theorem in Section 5 .

Remark. (i) In Theorem 2.1, $\nabla$ denotes the gradient in usual sense; $\partial$ indicates the Clarke generalized gradient; $N_{C}$ and $N_{U(t)}$ are the Clarke normal cones associated with $C$ and $U(t)$, respectively; $\Lambda_{i}^{\circ}, L_{i}^{\circ}, h_{i}^{\circ}$ are the Clarke generalized directional derivative; $T_{C}\left(x_{*}(0)\right)$ and $T_{U(t)}$ are the Clarke tangent cones associated with $C$ and $U(t)$, respectively (see Clarke (1983)).

(ii) To prove Theorem 2.1, we will follow the idea in the proof of Theorem 3.2.6 in Clarke (1983). By the analogous way, Ye and $\mathrm{Zhu}$ (1997) showed necessary optimality conditions for a bilevel perturbed differential inclusion problem (Theorem 3.1). However, in the proof of Theorem 3.1 they used a lemma (Lemma 6.2) whose proof seems insufficient. It seems that their Theorem 3.1 has not been proved. 


\subsection{Sufficient condition in a convex case}

Pinho and Vinter (1995) pointed out that for the nonsmooth optimal control problem without mixed state-control constraints, the weak maximum principle is sufficient for optimality in 'normal and convex' case, while the general form of the nonsmooth maximum principle may fail to be sufficient. For (NOCP), we show that the necessary optimality conditions in Theorem 2.1 are also sufficient in a 'convex' case. These consequences can be used in the multilevel optimization problems.

Now, let us denote by $(L O C P)$ the problem $(N O C P)$ whose state equation is given by

$$
\dot{x}(t)=A(t) x(t)+B(t) u(t)+b(t)
$$

where $A(\cdot):[0,1] \rightarrow R^{m \times m}$ and $B(\cdot):[0,1] \rightarrow R^{m \times n}$ are integrable, $b(\cdot):[0,1] \rightarrow R^{m}$ is measurable.

We will impose the following hypotheses:

(H1): $C$ is convex set of $R^{m}$.

(H2): $U(t)$ is convex in $R^{n}$ for almost all $t \in[0,1]$.

(H3): $L_{i}(i=0, \cdots, k)$ are Lebesgue measurable in $t$, convex in $(x, u)$.

(H4): $h_{i}(i=0, \cdots, k)$ are convex functions.

(H5): The functionals $\Lambda_{i}(i=1, \cdots, k)$ are concave.

Then, we have the following result:

THEOREM 2.2. Let $\left(x_{*}(\cdot), u_{*}(\cdot)\right)$ be an admissible process for (LOCP). Suppose that (H1)-(H5) hold. If there exist $p(\cdot) \in A C\left([0,1], R^{n}\right), \zeta(\cdot) \in L^{\infty}\left([0,1], R^{m}\right), \lambda_{i} \geq 0$ $(i=1, \cdots, k)$ and $\lambda_{0}>0$ such that conditions (2.1)-(2.4) are satisfied, then $\left(x_{*}(\cdot), u_{*}(\cdot)\right)$ is a minimizer for (LOCP).

REMARK. In Theorem 2.2, the notations $\partial$ and $N$ stand for the standard subdifferential and normal cone in the sense of convex analysis, respectively. The condition (2.2) is understood as

$$
\zeta=\zeta_{1}+\zeta_{2}, \quad \zeta_{1} \in \sum_{i=1}^{k} \lambda_{i} \partial\left(-\Lambda_{i}\left(u_{*}\right)\right), \quad \zeta_{2}(t) \in N_{U(t)}\left(u_{*}(t)\right) \text { a.e.. }
$$

Proof. In this convex case, the condition (2.1) implies that

$$
(\dot{p}(t)+p(t) A(t),-\zeta(t)+p(t) B(t)) \in \sum_{i=1}^{k} \lambda_{i} \partial L_{i}[t]
$$

Comparing the cost value of an arbitrary admissible process $(x(\cdot), u(\cdot))$ with that 
of $\left(x_{*}(\cdot), u_{*}(\cdot)\right)$, and by $(2.4)$ we see that

$$
\begin{aligned}
& \int_{0}^{1} L_{0}(t, x, u) d t+h_{0}(x(1))-\int_{0}^{1} L_{0}[t] d t-h_{0}\left(x_{*}(1)\right) \\
\geq & \frac{1}{\lambda_{0}}\left\{\sum_{i=1}^{k} \lambda_{i} \int_{0}^{1}\left(L_{i}(t, x, u)-L_{i}[t]\right) d t+\sum_{i=1}^{k} \lambda_{i}\left(h_{i}(x(1))-h_{i}\left(x_{*}(1)\right)\right)\right. \\
& \left.-\sum_{i=1}^{k} \lambda_{i}\left(\Lambda_{i}(u)-\Lambda_{i}\left(u_{*}\right)\right)+\int_{0}^{1}\left(p(\dot{x}-A x-B u-b)-p\left(\dot{x}_{*}-A x_{*}-B u_{*}-b\right)\right) d t\right\} \\
= & \frac{1}{\lambda_{0}}\left\{\sum_{i=1}^{k} \lambda_{i} \int_{0}^{1}\left(L_{i}(t, x, u)-L_{i}[t]\right) d t-\int_{0}^{1}(\dot{p}+p A)\left(x-x_{*}\right) d t\right. \\
& -\int_{0}^{1}(p B-\zeta)\left(u-u_{*}\right) d t+\sum_{i=1}^{k} \lambda_{i}\left(h_{i}(x(1))-h_{i}\left(x_{*}(1)\right)\right)+p(1)\left(x(1)-x_{*}(1)\right) \\
& +\sum_{i=1}^{k} \lambda_{i}\left(-\Lambda_{i}(u)+\Lambda_{i}\left(u_{*}\right)\right)-\int_{0}^{1} \zeta_{1}\left(u-u_{*}\right) d t \\
& \left.-p(0)\left(x(0)-x_{*}(0)\right)-\int_{0}^{1} \zeta_{2}\left(u-u_{*}\right) d t\right\} \\
=: & \Delta^{*} .
\end{aligned}
$$

Recall the definitions of subdifferential of convex functions and normal cone of convex sets. From (2.3), (2.7) and (2.8), it follows that $\Delta^{*} \geq 0$. Thus, $\left(x_{*}, u_{*}\right)$ is a minimizer for $(N O C P)$.

\section{Value Function}

The value functions $V_{1}(u)$ and $V_{2}(u, v)$ of (TDOP) may be nonsmooth even if all functions in $(D)$ are smooth. In such case, it is difficult to calculate their Clarke generalized gradients. On the differentiability, we recall the recent results given by Ye and $\mathrm{Zhu}$ (1997). To discuss the sufficient optimality conditions for trilevel optimization problems, we will also observe the convexity of the value functions under some assumptions.

For $\left(\varphi, G_{1}\right):[0,1] \times R^{m_{2}} \times R^{n_{1}} \times R^{n_{2}} \rightarrow R^{m_{2}} \times R$ and $\left(\psi_{;} G_{2}\right):[0,1] \times R^{m_{3}} \times R^{n_{1}}$ $\times R^{n_{2}} \times R^{n_{3}} \rightarrow R^{m_{2}} \times R$, we set

$$
\begin{aligned}
& \varphi^{\dagger}(t, y, u):=\left\{\left(\varphi(t, y, u, v), G_{1}(t, y, u, v)\right): v \in U_{2}(t)\right\} \\
& :[0,1] \times R^{m_{2}} \times R^{n_{1}} \rightarrow 2^{R^{m_{2}} \times R} \\
& H_{1}\left(t, y, p_{1}, u\right):=\sup _{v \in U_{2}(t)}\left\{\left\langle p_{1}, \varphi(t, y, u, v)\right\rangle-G_{1}(t, y, u, v)\right\} \\
& :[0,1] \times R^{m_{2}} \times R^{m_{2}} \times R^{n_{1}} \rightarrow R \\
& \psi^{\dagger}(t, z, u, v):=\left\{\left(v(t, z, u, v, w), G_{2}(t, z, u, v, w)\right): w \in U_{3}(t)\right\} \\
& :[0,1] \times R^{m_{3}} \times R^{n_{1}} \times R^{n_{2}} \rightarrow 2^{R^{n_{3}} \times R} \\
& H_{2}\left(t, z, p_{2}, u, v\right):=\sup _{w \in L_{3}(t)}\left\{<p_{2}, \dot{v}(t, z, u, v, w)>-G_{2}(t, z, u, v, w)\right\} \\
& :[0,1] \times R^{m_{3}} \times R^{m_{3}} \times R^{n_{1}} \times R^{n_{2}} \rightarrow R .
\end{aligned}
$$


To use the results in Ye and Zhu (1997), we assume (A7)-(A8) below.

(AT): $\left(G_{1}(t, y, u, v), \varphi(t, y, u, v), \nu^{t}(t, y, u), H_{1}\left(t, y, p_{1}, u\right)\right)\left(\left(t, y, p_{1}, u, v\right) \in[0,1] \times R^{m_{2}} \times\right.$ $R^{m_{2}} \times R^{n_{1}} \times R^{n_{2}}$ ) satisfies the following a)-d).

a) The functions $G$ and $\varphi$ are measurable in $t$ and continuous in $(y, u, v)$. The multifunction $\varphi^{\dagger}$ has nonempty, compact, convex values.

b) There exists a nonnegative function $\theta_{1}(t) \in L^{\infty}$, such that for almost all $t \in[0,1]$,

$$
\varphi^{\dagger}\left(t, y_{1}, u_{1}\right) \subset \varphi^{\dagger}\left(t, y_{2}, u_{2}\right)+\theta_{1}(t)\left(\left|y_{1}-y_{2}\right|+\left|u_{1}-u_{2}\right|\right) B_{R^{n_{2}}} .
$$

for any $y_{1}, y_{2} \in R^{m_{2}} ; u_{1}, u_{2} \in R^{n_{1}}$.

c) For each $u(\cdot) \in \mathcal{U}_{1}:=\left\{u(\cdot) \in L^{1}: u(t) \in U_{1}(t)\right.$ a.e. $\}$, there exists a nonnegative function $\rho_{u}(\cdot) \in L^{1}[0,1]$, such that $\varphi^{\dagger}(t, y, u(t)) \subset \rho_{u}(t) B$ for any $y \in R^{m_{2}} \quad$ a.e..

d) The partial Clarke generalized gradients $\partial_{\left(y, p_{1}\right)} H_{1}\left(t, y, p_{1}, u\right)$ and $\partial_{u} H_{1}\left(t, y, p_{1}, u\right)$ are upper semicontinuous with respect to $\left(t, y, p_{1}, u\right)$.

(A8): a)-d) above hold with $\left(G_{1}(t, y, u, v), \varphi(t, y, u, v), \varphi^{\dagger}(t, y, u), H_{1}\left(t, y, p_{1}, u\right)\right)$ replaced by $\left(G_{2}(t, z, \bar{u}, w), \psi(t, z, \bar{u}, w), \psi^{\dagger}(t, z, \bar{u}), H_{2}\left(t, z, p_{2}, \bar{u}\right)\right)\left(\bar{u}=(u, v),\left(t, z, p_{2}, \bar{u}, w\right) \epsilon\right.$ $\left.[0,1] \times R^{m_{3}} \times R^{m_{3}} \times R^{n_{1}+n_{2}} \times R^{n_{3}}\right)$.

Let $\gamma_{1}(t):=\int_{0}^{t} G_{1}(t, y(t), u(t), v(t)) d t$. Under the assumptions in Lemma 3.1 given below, according to the Filippov's Lemma (see Loewen (1993)), the optimal control problem $P_{B}[u]$ can be expressed by the following perturbed optimization problem with differential inclusion constraints:

$$
\begin{aligned}
\text { Minimize: } & g(y(1))+\gamma_{1}(1) \\
\text { subject to: } & \left(\dot{y}(t), \dot{\gamma}_{1}(t)\right) \in \varphi^{\dagger}(t, y(t), u(t)) \quad \text { a.e. } \\
& y(0) \in C_{2} .
\end{aligned}
$$

Recall a well-known result: if $y$ is an optimal state (corresponding to an optimal control $v$ ) for $P_{B}[u]$, then there exists a Hamiltonian multiplier $p_{1} \in A C$ with $\left(-\dot{p}_{1}(t), \dot{y}(t)\right) \in \partial_{\left(y, p_{1}\right)} H_{1}\left(t, y(t), p_{1}(t), u(t)\right) \quad$ a.e., $p_{1}(0) \in N_{C_{2}}(y(0))$ and $-p_{1}(1) \in$ $\partial g_{1}(y(1))$ (see Clarke (1975)).

We put

$$
\begin{gathered}
S_{u}:=\left\{y \in A C: y \text { is an optimal state for } P_{B}[u]\right\} \\
M_{u}(y):=\left\{p_{1} \in A C:\left(-\dot{p}_{1}(t), \dot{y}(t)\right) \in \partial_{\left(y, p_{1}\right)} H_{1}\left(t, y(t), p_{1}(t), u(t)\right\}\right. \text { a.e., } \\
\left.p_{1}(0) \in N_{C_{2}}(y(0)),-p_{1}(1) \in \partial g_{1}(y(1))\right\} \\
\partial_{u} H_{1}\left(\cdot, y(\cdot), p_{1}(\cdot), u(\cdot)\right):=\left\{\zeta \in L^{\infty} ; \zeta(t) \in \partial_{u} H_{1}\left\{t, y(t), p_{1}(t), u(t)\right) \text { a.e }\right\} .
\end{gathered}
$$

Thus, using the results of $\mathrm{Ye}$ and $\mathrm{Zhu}$ (1997), we obtain

LEMMA 3.1. Assume that $g_{1}$ is locally Lipschitz continuous, (A1) and (A2) hold for $C=C_{2}$ and $U(t)=U_{2}(t)$, respectively, and $(A 7)$ is satisfed. Then, $V_{1}(u)$ is locally Lipschitz continuous and.

$$
-\partial V_{1}(u) \subset c l^{*} \operatorname{co}\left\{\partial_{u} H_{1}\left(\cdot, y(\cdot), p_{1}(\cdot), u(\cdot)\right): \quad y \in S_{u}, \quad p_{1} \in M_{u}(y)\right\},
$$

where $\mathrm{cl}^{*}$ co denotes the weak convex closure. 
Similarly, for the value function $V_{2}(u, v)$ we have

LEMMA 3.2. In addition to (A8), assume that $g_{2}$ is locally Lipschitz continuous, (A1) and (A2) hold for $C=C_{2}$ and $U(t)=L_{3}(t)$, respectively. Then, $V_{2}(u, v)$ is locally Lipschitz continuous and

$$
-\partial V_{2}(u, v) \subset c l^{*} c o\left\{\partial_{(u, v)} H_{2}\left(\cdot, z(\cdot), p_{2}(\cdot), u(\cdot), v(\cdot)\right): z \in S_{(u, v)}, \quad p_{2} \in M_{(u, v)}(z)\right\},
$$

where

$$
\begin{gathered}
\partial_{(u, v)} H_{2}\left(\cdot, z(\cdot), p_{2}(\cdot), u(\cdot), v(\cdot)\right):=\left\{\zeta \in L^{\infty} ; \zeta(t) \in \partial_{(u, v)} H_{2}\left(t, z(t), p_{2}(t), u(t), v(t)\right\} \text { a.e. }\right\}, \\
S_{(u, v)}:=\left\{z \in A C: z \text { is an optimal state of } P_{C}[u, v]\right\} \\
M_{(u, v)}(z):=\left\{p_{2} \in A C:\left(-\dot{p}_{2}(t), \dot{z}(t)\right) \in \partial_{\left(z, p_{2}\right)} H_{2}\left(t, z(t), p_{2}(t), u(t), v(t)\right)\right. \text { a.e. } \\
\left.p_{1}(0) \in N_{C_{2}}(y(0)),-p_{1}(1) \in \partial g_{1}(y(1))\right\}
\end{gathered}
$$

Next, we proceed to the convexity of the following value function:

$$
\begin{array}{r}
V_{*}(u):=\inf \left\{\int_{0}^{1} G(t, y(t), u(t), v(t)) d t+g(y(1)): \dot{y}(t)=A(t) y(t)+B(t) u(t)+\right. \\
D(t) v(t)+b(t) \text { a.e, } y(0) \in C, v(t) \in U(t) \text { a.e. }\},
\end{array}
$$

where $(y, u, v) \in A C\left([0,1], R^{m}\right) \times L^{1}\left([0,1]: R^{n_{1}}\right) \times L^{1}\left([0,1], R^{n_{2}}\right), G:[0,1] \times R^{m} \times$ $R^{n_{1}} \times R^{n_{2}} \rightarrow R, g: R^{m} \rightarrow R, C \subset R^{m}, U(\cdot):[0,1] \rightarrow 2^{R^{n_{2}}}, A(\cdot):[0,1] \rightarrow R^{m \times m}$, $B(\cdot):[0,1] \rightarrow R^{m \times n_{1}}, D(\cdot):[0,1] \rightarrow R^{m \times n_{2}}$ and $b(\cdot):[0,1] \rightarrow R^{m}$.

LEMMA 3.3. Suppose that (H1) and (H2) are satisfied, $A(\cdot)$ is integrable, $B(\cdot)$, $D(\cdot), b(\cdot), G(\cdot, y, u, v)$ are measurable, then the following statements hold.

(i) If $G(t, \cdot, \cdot, \cdot)$ and $g(\cdot)$ are convex, then $V_{*}(u)$ is convex.

(ii) If $G(t, \cdot, \cdot, v)$ and $g(\cdot)$ are concave, then $V_{*}(u)$ is concave.

Proof. Let $u_{1}, u_{2}$ be arbitrary elements of $L^{1}[0,1]$, and put $\bar{u}=\lambda u_{1}+(1-\lambda) u_{2}$ for any $\lambda \in(0,1)$. We define

$$
\begin{gathered}
N(u):=\left\{(y, v) \in A C \times L^{1}: \dot{y}=A y+B u+D v+b \quad \text { a.e., } y(0) \in C, v(t) \in U(t) \text { a.e. }\right\}, \\
\Theta(y, u, v):=\int_{0}^{1} G(t, y(t), u(t), v(t)) d t+g(y(1)) .
\end{gathered}
$$

Then, we have $V_{*}(u)=\inf _{(y, v) \in N(u)} \Theta(y, u, v)$.

(i) If $N\left(u_{1}\right)$ or $N\left(u_{2}\right)$ is empty, then $\lambda V_{*}\left(u_{1}\right)+(1-\lambda) V_{*}\left(u_{2}\right)=+\infty \geq V_{*}(\bar{u})$. Thus we may assume that both $N\left(u_{1}\right)$ and $N\left(u_{2}\right)$ are not empty. For any $\left(y_{1}, v_{1}\right) \in N\left(u_{1}\right)$, $\left(y_{2}, v_{2}\right) \in N\left(u_{2}\right)$, let

$$
\bar{y}=\lambda y_{1}+(1-\lambda) y_{2}, \vec{v}=\lambda v_{1}+(1-\lambda) v_{2}
$$


It is easy to see that $(\bar{y}, \bar{v}) \in Y(\bar{u})$. Noting that $\Theta$ is convex, we see

$$
\lambda \Theta\left(y_{1}, u_{1}, v_{1}\right)+(1-\lambda) \Theta\left(y_{2}, u_{2}, v_{2}\right) \geq \Theta(\bar{y}, \bar{u}, \bar{v}) \geq \inf _{(y, v) \in N(\bar{u})} \Theta(y, \bar{u}, v) .
$$

Hence, we have

$$
\begin{aligned}
\lambda V_{*}\left(u_{1}\right)+(1-\lambda) V_{*}\left(u_{2}\right) & =\lambda \inf _{(y, v) \in N\left(u_{1}\right)} \Theta\left(y, u_{1}, v\right)+(1-\lambda) \inf _{(y, v) \in N\left(u_{2}\right)} \Theta\left(y, u_{2}, v\right) \\
& \geq \inf _{(y, v) \in N(\bar{u})} \Theta(y, \bar{u}, v)=V_{*}(\bar{u}) .
\end{aligned}
$$

(ii) If $N(\bar{u})=\emptyset$, then $V_{*}(\bar{u})=+\infty \geq \lambda V_{*}\left(u_{1}\right)+(1-\lambda) V_{*}\left(u_{2}\right)$. Thus, we may assume $N(\bar{u}) \neq \emptyset$. For every $(\tilde{y}, \tilde{v}) \in N(\bar{u})$, let $\tilde{y}_{i}$ be a solution of following equation,

$$
\left\{\begin{array}{l}
\dot{y}(t)=A(t) y(t)+B(t) u_{i}(t)+D(t) \tilde{v}(t)+b(t) \quad \text { a.e } \quad(i=1,2) \\
y(0)=\tilde{y}(0)
\end{array}\right.
$$

Then, we have

$$
\bar{y}=\lambda \bar{y}_{1}+(1-\lambda) \tilde{y}_{2},\left(\tilde{y}_{i}, \tilde{v}\right) \in N\left(u_{i}\right)(i=1,2) .
$$

By concavity of $G$ and $g$, we see that

$$
\begin{aligned}
\Theta(\bar{y}, \bar{u}, \bar{v}) & \geq \lambda \Theta\left(\bar{y}_{1}, u_{1}, \bar{v}\right)+(1-\lambda) \Theta\left(\bar{y}_{2}, u_{2}, \bar{v}\right) \\
& \geq \lambda \inf _{(y, v) \in N\left(u_{1}\right)} \Theta\left(y, u_{1}, v\right)+(1-\lambda) \inf _{(y, v) \in N\left(u_{2}\right)} \Theta\left(y, u_{2}, v\right),
\end{aligned}
$$

which implies that

$$
\begin{aligned}
V_{*}(\bar{u})=\inf _{(y, v) \in N(\bar{u})} \Theta(y, \bar{u}, v) & \geq \lambda \inf _{(y, v) \in N\left(u_{1}\right)} \Theta\left(y, u_{1}, v\right)+(1-\lambda) \inf _{(y, v) \in N\left(u_{2}\right)} \Theta\left(y, u_{2}, v\right) \\
& \geq \lambda V_{*}\left(u_{1}\right)+(1-\lambda) V_{*}\left(u_{2}\right) .
\end{aligned}
$$

We have therefore proved this lemma.

\section{Optimality Conditions for (TDOP)}

In this section we derive the optimality conditions for the trilevel dynamic optimization problem (TDOP). A simple example will be given in Section 5 .

In Theorem 4.1 and Corollary 4.2 given below, $\partial$ indicates the Clarke generalized gradient and $N_{C_{i}}, N_{U_{i}(t)}(i=1,2.3)$ denote the Clarke normal cones; while in Theorem 4.3 , these stand for the subdifferential and the normal cones in the sense of convex analysis, respectively.

First we derive necessary optimality conditions for (TDOP).

Let $\left(x_{*}, y_{*} ; z_{*} ; u_{*}, v_{*}, w_{*}\right)=\left(\bar{x}_{*} ; \bar{u}_{*}\right)$ be a local optimal solution of the trilevel optimality problem $(T D O P)$, i.e. $\left(\tilde{x}_{*}: \tilde{u}_{*}\right)$ is a local minimizer for the optimal control 
problem $P$. Notice that $P$ can be easily written in the form (NOCP) with

$$
\begin{aligned}
& \tilde{x}(\cdot):=(x(\cdot), y(\cdot), z(\cdot)) \in A C\left([0,1], R^{m}\right), \quad m=m_{1}+m_{2}+m_{3} \\
& \bar{u}(\cdot):=(u(\cdot), v(\cdot), w(\cdot)) \in L^{1}\left([0,1], R^{n}\right), \quad n=n_{1}+n_{2}+n_{3} \\
& C:=C_{1} \times C_{2} \times C_{3}, \quad U(t):=U_{1}(t) \times U_{2}(t) \times U_{3}(t) \\
&\left(D^{*}\right): \quad \\
& \Phi(t, \tilde{x}, \tilde{u}):=(\phi(t, x, \tilde{u}), \vartheta(t, y, u, v), \psi(t, z, \bar{u})) \\
& L_{0}(t, \bar{x}, \bar{u}):=F(t, x, \tilde{u}), \quad h_{0}(\tilde{x}(0)):=f(x(0)) \\
& L_{1}(t, \tilde{x}, \tilde{u}):=G_{1}(t, y, u, v), \quad h_{1}(\tilde{x}(0)):=g_{1}(y(0)) \\
& L_{2}(t, \tilde{x}, \tilde{u}):=G_{2}(t, z, \tilde{u}), \quad h_{2}(\tilde{x}(0)):=g_{2}(z\{0)) \\
& \Lambda_{1}(\tilde{u}):=V_{1}(u), \quad \Lambda_{2}(\tilde{u}):=V_{2}(u, v) .
\end{aligned}
$$

If the assumptions in Theorem 2.1 are satisfied, then there exist an absolutely continuous function $p(\cdot):[0,1] \rightarrow R^{m_{1}} \times R^{m_{2}} \times R^{m_{3}}$, a measurable essentially bounded function $\zeta(\cdot):[0,1] \rightarrow R^{n_{1}} \times R^{n_{2}} \times R^{n_{3}}$ and $\lambda_{0}, \lambda_{1}, \lambda_{2} \geq 0$ with $\sum_{i=0}^{2} \lambda_{i}=1$, such that

$$
\begin{gathered}
(-\dot{p}(t), \zeta(t)) \in \nabla_{(\bar{x}, \tilde{u})} H\left(t, \tilde{x}_{*}, \tilde{u}_{*}\right)-\partial_{(\tilde{x}, \tilde{u})} E\left(t, \tilde{x}_{*}, \tilde{u}_{*}\right) \\
\zeta=\mu_{1}+\mu_{2},-\mu_{1} \in \lambda_{1} \partial_{\bar{u}} V_{1}\left(u_{*}\right)+\lambda_{2} \partial_{\bar{u}} V_{2}\left(u_{*}, v_{*}\right), \mu_{2}(t) \in N_{U(t)}\left(\tilde{u}_{*}(t)\right) \quad \text { a.e. } \\
p(0) \in N_{C}\left(\bar{x}_{*}(0)\right),-p(1) \in \lambda_{0} \partial_{\bar{x}} f\left(x_{*}(1)\right)+\lambda_{1} \partial_{\tilde{x}} g_{1}\left(y_{*}(1)\right)+\lambda_{2} \partial_{\bar{x}} g_{2}\left(z_{*}(1)\right)
\end{gathered}
$$

Here, we set

$$
\begin{gathered}
H(t, \tilde{x}, \tilde{u})):=\left\langle p_{1}, \phi(t, x, u, v, w)\right\rangle+\left\langle p_{2}, \varphi(t, y, u, v)\right\rangle+\left\langle p_{3}, \psi(t, z, u, v, w)\right\rangle \\
E(t, \tilde{x}, \tilde{u}):=\lambda_{0} F(t, x, u, v, w)+\lambda_{1} G_{1}(t, y, u, v)+\lambda_{2} G_{2}(t, z, u, v, w) .
\end{gathered}
$$

Now we arrive at the following conclusion.

THEOREM 4.1. Suppose that $\left(x_{*}, y_{*}, z_{*} ; u_{*}, v_{*}, w_{*}\right)$ is a local minimizer for (TDOP). Let assumptions (A1)-(A6) hold for the data $\left\{C, U(t), \Phi, L_{i}, h_{i}(i=0,1,2), \Lambda_{i}\right.$ $(i=1,2)\}$ in $\left(D^{*}\right)$ associated with $\left(\bar{x}_{*} ; \bar{u}_{*}\right)$. Then,

(i) there exist $p(\cdot)=\left(p_{1}(\cdot), p_{2}(\cdot), p_{3}(\cdot)\right) \in A C, \zeta(\cdot)=\left(\zeta_{1}(\cdot), \zeta_{2}(\cdot), \zeta_{3}(\cdot)\right) \in L^{\infty}$ and $\lambda_{0}, \lambda_{1}, \lambda_{2} \geq 0$ with $\sum_{i=0}^{2} \lambda_{i}=1$, such that (4.1) and the following (4.2)-(4.5) hold.

$$
\begin{gathered}
\left(\zeta_{1}, \zeta_{2}, \zeta_{3}\right)=\left(\bar{\zeta}_{1}+\hat{\zeta}_{1}+\tilde{\zeta}_{1}, \hat{\zeta}_{2}+\bar{\zeta}_{2}, \zeta_{3}\right) \\
-\bar{\zeta}_{1} \in \lambda_{1} \partial_{u} V_{1}\left(u_{*}\right),-\left(\tilde{\zeta}_{1}, \hat{\zeta}_{2}\right) \in \lambda_{2} \partial_{(u, v)} V_{2}\left(u_{*}, v_{*}\right) \\
\left(\tilde{\zeta}_{1}, \tilde{\zeta}_{2}, \zeta_{3}\right) \in N_{U_{1}(t)}\left(u_{*}(t)\right) \times N_{U_{2}(t)}\left(v_{*}(t)\right) \times N_{U_{3}(t)}\left(w_{*}(t)\right) \quad \text { a.e. } \\
\left(p_{1}(0), p_{2}(0), p_{3}(0)\right) \in N_{C_{1}}\left(x_{*}(0)\right) \times N_{C_{2}}\left(y_{*}(0)\right) \times N_{C_{3}}\left(z_{*}(0)\right) \\
-\left(p_{1}(1), p_{2}(1), p_{3}(1)\right\} \in \lambda_{0} \partial_{x} f\left(x_{*}(1)\right) \times \lambda_{1} \partial_{y} g_{1}\left(y_{*}(1)\right) \times \lambda_{1} \partial_{z} g_{2}\left(z_{*}(1)\right) .
\end{gathered}
$$

(ii) If the constraint qualifications (2.5) and (2.6) in Theorem 2.1 hold for the data $\left\{\Lambda_{i}, L_{i}, h_{i}(i=1,2), \Phi, C, U(t)\right\}$ in $\left(D^{*}\right)$, then $\lambda_{0}>0$. 
(iii) If $F, G_{1}, G_{2}$ are convex in $(\tilde{x} ; \tilde{u})$, then (4.1) implies that

$$
\begin{aligned}
-\dot{p}_{1}(t) \in p_{1}(t)-\nabla_{x} \phi\left(t, x_{*}(t), \tilde{u}_{*}(t)\right)-\lambda_{0} \partial_{x} F\left(t, x_{*}(t), \tilde{u}_{*}(t)\right) \\
-\dot{p}_{2}(t) \in p_{2}(t) \cdot \nabla_{y} \rho\left(t, y_{*}(t), u_{*}(t), v_{*}(t)\right)-\lambda_{1} \partial_{y} G_{1}\left(t, y_{*}(t), u_{*}(t), v_{*}(t)\right) \\
-\dot{p}_{3}(t) \in p_{3}(t) \cdot \nabla_{z} \psi\left(t, z_{*}(t), \tilde{u}_{*}(t)\right)-\lambda_{2} \partial_{z} G_{2}\left(t, z_{*}(t), \bar{u}_{*}(t)\right) \\
\zeta_{1}(t) \in \nabla_{u} H\left(t, \bar{x}_{*}(t), \bar{u}_{*}(t)\right)-\partial_{u} E\left(t, \tilde{x}_{*}(t), \tilde{u}_{*}(t)\right) \\
\zeta_{2}(t) \in \nabla_{u} H\left(t_{*} \tilde{x}_{*}(t), \bar{u}_{*}(t)\right)-\partial_{\tau^{\prime}} E\left(t, \tilde{x}_{*}(t), \tilde{u}_{*}(t)\right) \\
\zeta_{3}(t) \in p_{1}(t) \cdot \nabla_{w} \phi\left(t, x_{*}(t), \tilde{u}_{*}(t)\right)+p_{3}(t) \cdot \nabla_{w} \psi\left(t, z_{*}(t), \bar{u}_{*}(t)\right) \\
-\lambda_{0} \partial_{u} F\left(t, x_{*}(t), \tilde{u}_{*}(t)\right)-\lambda_{2} \partial_{w} G_{2}\left(t, z_{*}(t), \tilde{u}_{*}(t)\right) .
\end{aligned}
$$

Combining Theorem 4.1, Lemma 3.1 and Lemma 3.2, we obtain

Corollary 4.2. Assume that $\left(x_{*}, y_{*}, z_{*} ; u_{*}, v_{*}, w_{*}\right)$ be a local minimizer for the (TDOP), the assumptions (A1)-(A5) hold for the data $\left\{C, U(t), \Phi, L_{i}, h_{i}, i=0,1,2\right\}$ in $\left(D^{*}\right)$ associated with $\left(\tilde{x}_{*} ; \tilde{u}_{*}\right)$. Let $(A 7)$ and $(A 8)$ hold. Then there exist $\left(p_{1}(\cdot), p_{2}(\cdot), p_{3}(\cdot)\right)$ $\in A C,\left(\zeta_{1}(\cdot), \zeta_{2}(\cdot), \zeta_{3}(\cdot)\right) \in L^{\infty}$ and $\lambda_{0}, \lambda_{1}, \lambda_{2} \geq 0$ with $\sum_{i=1}^{2} \lambda_{i}=1$, such that (4.1), (4.3)-(4.5) and the following (4.6) hold.

$$
\begin{aligned}
& \left(\zeta_{1}, \zeta_{2}, \zeta_{3}\right)=\left(\bar{\zeta}_{1}+\hat{\zeta}_{1}+\tilde{\zeta}_{1}, \hat{\zeta}_{2}+\tilde{\zeta}_{2}, \zeta_{3}\right) \\
& -\bar{\zeta}_{1} \in c l^{*} \operatorname{co}\left\{\partial_{u} H_{1}\left(\cdot, \alpha(\cdot), q(\cdot), u_{*}(\cdot)\right): \alpha \in S_{u_{*}}, q \in M_{u_{*}}(\alpha)\right\} \\
& -\left\{\hat{\zeta}_{1}, \hat{\zeta}_{2}\right) \in c l^{*} \operatorname{co}\left\{\partial_{(u, v)} H_{2}\left(\cdot, \alpha(\cdot), q(\cdot), u_{*}(\cdot), v_{*}(\cdot)\right): \alpha \in S_{\left(u_{*}, v_{*}\right)}, q \in M_{\left(u_{*}, v_{*}\right)}(\alpha)\right\}
\end{aligned}
$$

Now we replace the state equations in $(T D O P)$ by linear systems

$$
\begin{array}{ll}
\dot{x}(t)=A_{1}(t) x(t)+B_{1}(t) u(t)+D_{1}(t) v(t)+E_{1}(t) w(t)+b_{1}(t) & \text { a.e. } \\
\dot{y}(t)=A_{2}(t) y(t)+B_{2}(t) u(t)+D_{2}(t) v(t)+b_{2}(t) & \text { a.e. } \\
\dot{z}(t)=A_{3}(t) z(t)+B_{3}(t) u(t)+D_{3}(t) v(t)+E_{3}(t) w(t)+b_{3}(t) & \text { a.e. }
\end{array}
$$

where $A_{i}(t):[0,1] \rightarrow R^{m_{i} \times m_{i}}, B_{i}(t):[0,1] \rightarrow R^{m_{i} \times n_{2}}, D_{i}(t):[0,1] \rightarrow R^{m_{i} \times n_{2}} \quad(i=$ $1,2,3)$ and $E_{i}(t):[0,1] \rightarrow R^{m_{i} \times n_{3}}(i=1,3)$ are integrable, $b_{i}(t):[0,1] \rightarrow R^{m_{i}}$ $(i=1,2,3)$ are measurable. We denote this problem by $\left(T D O P^{*}\right)$.

Notice that the condition (2.4) always hold for $P$. From Theorem 2.2 and Lemma 3.3 we get directly the following sufficient optimality conditions for $\left(T D O P^{*}\right)$.

THEOREM 4.3. Assume that the control $\left(u_{*}, v_{*}, w_{*}\right)$ corresponding to $\left(x_{*}, y_{*}, z_{*}\right)$ is admissible for $\left(T D O P^{*}\right)$. Let the data $\left\{C, U(t), L_{i}, i=0,1,2\right\}$ in $\left(D^{*}\right)$ satisfy (H1)(H3), respectively, $G_{1}(t, \cdot \cdot, v), G_{2}(t, \cdot, \cdot, \cdot, w)$ be concave, and $g_{1}(\cdot), g_{2}(\cdot)$ be affine. If there exist $\left(p_{1}, p_{2}, p_{3}\right)(\cdot) \in A C,\left(\zeta_{1}, \zeta_{2}, \zeta_{3}\right)(\cdot) \in L^{\infty}$ and $\lambda_{0}>0, \lambda_{1}, \lambda_{2} \geq 0$ such that (4.1)-(4.5) hold, then $\left(x_{*}, y_{*}, z_{*} ; u_{*}, v_{*}, w_{*}\right)$ is an optimal solution of (TDOP*).

REMARK. For the trilevel optimization problem (TDOP), sometimes, the player $A$ has to guarantee that the costs of the players $B$ and $C$ are not too large. The problem 
$P_{A}$ of $(T O C P)$ with such constraints is stated as follows,

$$
\begin{aligned}
\text { Minimize : } & \int_{0}^{1} F(t, x(t), u(t), v(t), w(t)) d t+f(x(1)\} \\
\text { subject to : } & x(t)=\phi(t, x(t), u(t), v(t), w(t)) \\
& x(0) \in C_{1} \\
& u(t) \in C_{1}(t) \quad \text { a.e. } \\
& v \in \mathcal{V}[u] \\
& w \in \mathcal{W}[u, v] \\
& \int_{0}^{1} G_{1}(t, y(t), u(t), v(t)) d t+g_{1}(y(1)) \leq M_{1} \\
& \int_{0}^{1} G_{2}(t, z(t), u(t), v(t), w(t)) d t+g_{2}(z(1)) \leq M_{2},
\end{aligned}
$$

where $M_{1}, M_{2}>0$. From the results in Section 2 and 3, we can also get optimality conditions for this trilevel optimization problem.

\section{Proof of Theorem 2.1}

Proof. Let us omit the variable $t$ when this does not cause confusion. We put

$$
\begin{gathered}
M:=\left\{(x, u) \in A C \times L^{1}: \dot{x}(t)=\Phi(t, x(t), u(t)) \text { a.e, } x(0) \in C, u(t) \in U^{\top}(t) \text { a.e. }\right\}, \\
\Delta\left(\left(x_{1}, u_{1}\right),\left(x_{2}, u_{2}\right)\right):=\left\|u_{1}-u_{2}\right\|_{L^{1}}+\left|x_{1}(0)-x_{2}(0)\right| .
\end{gathered}
$$

It is evident that $\Delta$ is a metric, and $M$ is a complete metric space with respect to $\Delta$.

For given $\epsilon>0$, Let

$$
\begin{gathered}
\Gamma_{\epsilon}(x, u):=\max \left\{\bar{G}_{1}(x, u), \cdots, \bar{G}_{k}(x, u), F(x, u)-F\left(x_{*}, u_{*}\right)+\epsilon^{2}\right\}, \\
F(x, u):=\int_{0}^{1} L_{0}(t, x(t), u(t)) d t+h_{0}(x(1)) .
\end{gathered}
$$

Then, we see that $\Gamma_{\epsilon}(x, u)>0$ for all $(x, u) \in M$. It follows that

$$
\Gamma_{\epsilon}\left(x_{*}, u_{*}\right) \leq \inf _{(x, u) \in M} \Gamma_{\epsilon}(x, u)+\epsilon^{2} .
$$

Thus, by the Ekeland Variational principle, we have

LEMMA 5.1. There is an element $\left(x_{0}, u_{0}\right) \in M$, such that $\left(x_{0}, u_{0}\right)$ minimizes

$$
\Gamma_{\epsilon}(x, u)+\epsilon \Delta\left((x, u),\left(x_{0}, u_{0}\right)\right)
$$

over all $(x, u) \in M$, and

$$
\Delta\left(\left(x_{m}, u_{m}\right),\left(x_{0}, u_{0}\right)\right) \leq \epsilon, \quad \Gamma_{\epsilon}\left(x_{0}, u_{0}\right) \leq \epsilon^{2} .
$$

The following Lemma 5.2 will be used to derive Lemma 5.3 . 
LEMMA 5.2. Let $\mathcal{U}=\left\{u \in L^{1}[0,1]: u(t) \in \mathcal{L}(t)\right.$ a.e. $\}$. If $u_{0}(\cdot) \in L^{1}$, then

$$
\int_{0}^{1} \inf _{v \in L^{*}(t)}\left|u_{0}(t)-v\right| d t=\inf _{u \in \mathcal{U}} \int_{0}^{1}\left|u_{0}(t)-u(t)\right| d t .
$$

In fact, by the measurability theorem (see Loewen (1993)), there is a sequence of Lebesgue measurable functions $v_{n}$ such that $U(t)=c l\left\{v_{n}(t): n=1,2, \cdots\right\}$.

such that

Let $\Psi(t)=\inf _{v \in Z^{(}(t)}\left|u_{0}(t)-v\right|$. For any $\epsilon^{\circ}>0$, there exist $v_{t} \in U(t)$ and $n_{0} \in N$,

$$
\Psi(t)+\epsilon^{\circ}>\left|u_{0}(t)-v_{0}(t)\right|,\left|v_{0}(t)-v_{n_{0}}(t)\right|<\epsilon^{\circ} .
$$

Then, we have

$$
\begin{aligned}
\inf _{n \in N}\left|u_{0}(t)-v_{n}(t)\right| & \geq \inf _{v \in U(t)}\left|u_{0}(t)-v\right|>\left|u_{0}(t)-v_{0}(t)\right|-\epsilon^{\circ} \geq\left|u_{0}(t)-v_{n_{0}}(t)\right|-2 \epsilon^{\circ} \\
& \geq \inf _{n \in N^{*}}\left|u_{0}(t)-v_{n}(t)\right|-2 \epsilon^{\circ}
\end{aligned}
$$

which implies that

$$
0 \leq \inf _{n \in N}\left|u_{0}(t)-v_{n}(t)\right|-\Psi(t) \leq 2 \epsilon^{\circ} .
$$

Thus, $\Psi(t)=\inf _{n \in N}\left|u_{0}(t)-v_{n}(t)\right|$. It is obvious that, for every $u(\cdot) \in \mathcal{U}$,

$$
\int_{0}^{1} \inf _{v \in U(t)}\left|u_{0}(t)-v\right| d t \leq \int_{0}^{1}\left|u_{0}(t)-u(t)\right| d t .
$$

For $\bar{\epsilon}>0$, consider a multifunction

$$
\Omega(t):=\left\{u \in U(t) ;\left|u_{0}(t)-u\right| \leq \inf _{v \in U(t)}\left|u_{0}(t)-v\right|+\frac{1}{2} \bar{\epsilon}\right\},
$$

which is measurable with closed nonempty values. By the Measurable selections Theorem (see Loewen (1993)), $\Omega$ admits a measurable selection $\bar{u}(\cdot)$. Since $\bar{u}$ is measurable and $\bar{u}(t) \in \Omega(t)$ a.e.. We see that $\bar{u}(\cdot) \in \mathcal{U}$, and

$$
\int_{0}^{1}\left|u_{0}(t)-\bar{u}(t)\right| d t<\int_{0}^{1} \inf _{v \in U(t)}\left|u_{0}(t)-v\right| d t+\bar{\epsilon} .
$$

Combining (5.3) and (5.4), we get (5.2).

LEMMA 5.3. The $\left(x_{0}, u_{0}\right)$ above is a local minimizer for the function.

$$
\begin{aligned}
\Lambda(x, u):= & \Gamma_{\epsilon}(x, u)+\epsilon \Delta\left((x, u),\left(x_{0}, u_{0}\right)\right)+K_{2} \int_{0}^{1}|\dot{x}(t)-\Phi(t, x(t), u(t))| d t \\
& +K_{1} d_{C}(x(0))+K_{1} \int_{0}^{1} d_{U(t)}(u(t)) d t,
\end{aligned}
$$

where $d_{C}(x(0)), d_{U(t)}(u(t))$ denote the distances of the points $x(0)$ and $u(t)$ to the sets $C$ and $U(t)$, respectively, and

$$
\begin{aligned}
& \left.K_{1}=\bar{K}\left(K K^{\prime}+1\right)+\epsilon\right), K_{2}=\bar{K} K \\
& \bar{K}=\max \left\{\left\|k_{L}\right\|_{L^{x}}+k_{h},\left\|k_{L}\right\|_{L^{\star}}+k_{\Lambda}\right\} \\
& K=\exp \int_{0}^{1} k(t) d t, \quad K^{\prime}=\|k\|_{L^{\infty}}
\end{aligned}
$$


Suppose this lemma to be false. Then, there exists a sequence $\left\{\left(x_{i}, u_{i}\right)\right\}$ converging to $\left(x_{0}, u_{0}\right)$ with $\Lambda\left(x_{i}, u_{i}\right)<\Lambda\left(x_{0}, u_{0}\right)$. Let $\lambda\left(x_{i}, u_{i}\right)=\Lambda\left(x_{0}, u_{0}\right)-2 \epsilon_{i} K_{1}$ (where $\left.\epsilon_{i}>0\right)$. By Lemma 5.2 there is $\tilde{u}_{i}(\cdot) \in \mathcal{U}$ such that

$$
\left\|\tilde{u}_{i}-u_{i}\right\|_{L^{1}} \leq \int_{0}^{1} \inf _{v \in L^{(}(t)}\left|u_{i}(t)-v\right| d t+\epsilon_{i} .
$$

Since $C$ is closed, there are $c_{i} \in C(i=0 \cdots k)$ such that $d_{C}\left(x_{i}(0)\right)=\left|x_{i}(0)-c_{i}\right|$. Let $\bar{x}_{i}(t)=x_{i}(t)-x_{i}(0)+c_{i}$, thus for sufficiently large $i$, it holds that

$$
\begin{aligned}
& \int_{0}^{1}\left|\dot{\bar{x}}_{i}(t)-\Phi\left(t, \bar{x}_{i}(t), \tilde{u}_{i}(t)\right)\right| d t \\
& \leq \int_{0}^{1}\left|\dot{x}_{i}(t)-\Phi\left(t, x_{i}(t), u_{i}(t)\right)\right| d t+K^{\prime}\left(\left|i \bar{u}_{i}-u_{i} \|_{L^{1}}+\right| x_{i}(0)-c_{i} \mid\right) .
\end{aligned}
$$

Let $\tilde{x}_{i}(t)$ be a solution of the following integral equation

$$
y(t)=\bar{x}_{i}(0)+\int_{0}^{t} \Phi\left(t, y(t), \tilde{u}_{i}(t)\right) d t .
$$

We see that $\left(\tilde{x}_{i}, \tilde{u}_{i}\right) \in M$ with $\tilde{x}_{i}(0)=\bar{x}_{i}(0)$ and

$$
\begin{aligned}
\left|\tilde{x}_{i}(t)-\bar{x}_{i}(t)\right| & \leq\left|\int_{0}^{t}\left(\Phi\left(t, \bar{x}_{i}, \bar{u}_{i}\right)-\dot{\bar{x}}_{i}\right) d t\right| \\
& \leq\left|\int_{0}^{t}\left(\Phi\left(t, \bar{x}_{i}, \tilde{u}_{i}\right)-\dot{\bar{x}}_{i}\right) d t\right|+\left|\int_{0}^{t}\left(\Phi\left(t, \tilde{x}_{i}, \tilde{u}_{i}\right)-\Phi\left(t, \bar{x}_{i}, \tilde{u}_{i}\right)\right) d t\right| \\
& \left.\leq \mid \int_{0}^{t} \Phi\left(t, \bar{x}_{i}, \bar{u}_{i}\right)-\dot{\bar{x}}_{i}\right) d t|+| \int_{0}^{t} k(t)\left|\tilde{x}_{i}(t)-\bar{x}_{i}(t)\right| d t \mid .
\end{aligned}
$$

It follows that

$$
\left\|\tilde{x}_{i}-\bar{x}_{i}\right\|_{L^{\times}} \leq K \int_{0}^{1}\left|\dot{\bar{x}}_{i}(t)-\Phi\left(t, \bar{x}_{i}(t), \tilde{u}_{i}(t)\right)\right| d t .
$$

(5.6) and (5.7) lead to

$$
\begin{aligned}
\left\|\tilde{x}_{i}-x_{i}\right\|_{L^{x}} \leq & K \int_{0}^{1}\left|\dot{x}_{i}(t)-\Phi\left(t, x_{i}(t), u_{i}(t)\right)\right| d t+K K^{\prime}\left\|\bar{u}_{i}-u_{i}\right\|_{L^{1}} \\
& +\left(K K^{\prime}+1\right)\left|x_{i}(0)-c_{i}\right|
\end{aligned}
$$

From (A3), (5.8) and (5.5), we have

$$
\begin{aligned}
& \Gamma_{\epsilon}\left(\tilde{x}_{i}, \tilde{u}_{i}\right)+\epsilon \Delta\left(\left(\tilde{x}_{i}, \tilde{u}_{i}\right),\left(x_{0}, u_{0}\right)\right) \\
\leq & \Gamma_{\epsilon}\left(x_{i}, u_{i}\right)+\epsilon \Delta\left(\left(x_{i}, u_{i}\right),\left(x_{0}, u_{0}\right)\right)+\bar{K}\left(\left\|\tilde{u}_{i}-u_{i}\right\|_{L^{1}}+\left\|\tilde{x}_{i}-x_{i}\right\|_{C}\right) \\
& +\epsilon\left(\left\|\tilde{u}_{i}-u_{i}\right\|_{L^{1}}+\left|x_{i}(0)-c_{i}\right|\right) \\
\leq & \Gamma_{\epsilon}\left(x_{i}, u_{i}\right)+\epsilon \Delta\left(\left(x_{i}, u_{i}\right),\left(x_{0}, u_{0}\right)\right)+\bar{K} K \int_{0}^{1}\left|\dot{x}_{i}(t)-\Phi\left(t, x_{i}(t), u_{i}(t)\right)\right| d t \\
& +\left(\bar{K}\left(K K^{\prime}+1\right)+\epsilon\right)\left(\int_{0}^{1} \inf _{v \in U(t)}\left|u_{i}(t)-v\right| d t+\epsilon_{i}+\left|x_{i}(0)-c_{i}\right|\right) \\
= & \Lambda\left(x_{i}, u_{i}\right)+\epsilon_{i} K_{1} \\
< & \Lambda\left(x_{0}, u_{0}\right) \\
= & \Gamma_{\epsilon}\left(x_{0}, u_{0}\right)
\end{aligned}
$$


which contradicts Lemma 5.1 , so Lemma 5.3 holds.

By Lemma 5.3, we know $0 \in \partial \mathrm{A}\left(x_{0}, u_{0}\right)$, i.e.

$$
\begin{aligned}
& 0 \in \partial \Gamma_{\epsilon}\left(x_{0}, u_{0}\right)+\partial \epsilon \Delta\left(\left(x_{0}, u_{0}\right),\left(x_{0}, u_{0}\right)\right)+\partial K_{1} d_{C}\left(x_{0}(0)\right)+ \\
& \quad \partial K_{1} \int_{0}^{1} d_{U(t)}\left(u_{0}(t)\right) d t+\partial K_{2} \int_{0}^{1}\left|\dot{x}_{0}(t)-\Phi\left(t, x_{0}(t), u_{0}(t)\right)\right| d t .
\end{aligned}
$$

According to the formulas of the generalized gradients (see Clarke (1983)), we have the following.

(a) For every $\xi \in \partial \Gamma_{\epsilon}\left(x_{0}, u_{0}\right)$, there exist functions $\xi_{i}, \eta_{i}(i=0, \cdots, k)$ with $\left(\xi_{i}, \eta_{i}\right)(t) \in$ $\partial L_{i}\left(t, x_{0}(t), u_{0}(t)\right)$ a.e. and $\nu_{i} \in \partial h_{i}\left(x_{0}(1)\right)(i=0, \cdots, k), \bar{\eta}_{i} \in-\partial \Lambda_{i}\left(u_{0}\right)(i=1, \cdots, k)$, $\bar{\lambda}_{i} \geq 0$ for $i \in I_{0}\left(x_{0}, u_{0}\right)$ with $\sum_{i \in I\left(x_{0}, u_{0}\right)} \bar{\lambda}_{i}=1$, such that for any $(x, u) \in A C \times L^{1}$

$$
\xi(x, u)=\sum_{i=0}^{k} \bar{\lambda}_{i} \int_{0}^{1}\left\{\left\langle\xi_{2}, x\right\rangle+\left\langle\eta_{i}, u\right\rangle\right\} d s+\sum_{i=0}^{k} \bar{\lambda}_{i}\left\langle\nu_{i}, x(1)\right\rangle+\sum_{i=1}^{k} \bar{\lambda}_{i} \int_{0}^{1}\left\langle\bar{\eta}_{i}, u\right\rangle d s
$$

where, $I_{0}\left(x_{0}, u_{0}\right):=\left\{i \in[0, \cdots, k]: \bar{G}_{i}\left(x_{0}, u_{0}\right)=\Gamma_{\epsilon}\left(x_{0}, u_{0}\right)\right\}, \bar{G}_{0}\left(x_{0}, u_{0}\right):=F(x, u)-$ $F\left(x_{*}, u_{*}\right)+\epsilon^{2}$, and $\bar{\lambda}_{i}:=0$ for $i \notin I\left(x_{0}, u_{0}\right)$.

(b) For every $\xi \in \partial \epsilon \Delta\left(\left(x_{0}, u_{0}\right),\left(x_{0}, u_{0}\right)\right)$, there are function $\theta_{2}$ with $\theta_{2}(t) \in \epsilon B_{R^{n}}$ a.e. and $\theta_{1} \in \epsilon B_{R^{\prime \prime}}$ such that for every $(x, u) \in A C \times L^{1}$

$$
\xi(x(0), u)=\left\langle\theta_{1}, x(0)\right\rangle+\int_{0}^{1}\left\langle\theta_{2}, u\right\rangle d s .
$$

(c) Every $\xi \in \partial K_{1} d_{C}\left(x_{0}(0)\right)$ corresponds to a mapping $r \in K_{1} \partial d_{C}\left(x_{0}(0)\right)$ with

$$
\xi(x)=\langle r, x(0)\rangle \quad \text { for any } x \in A C .
$$

(d) For every $\xi \in \partial K_{1} \int_{0}^{1} d_{U(t)}\left(u_{0}(t)\right) d t$, there is a function $\bar{\eta}$ with $\bar{\eta}(t) \in K_{1} \partial d_{U(t)}\left(u_{0}(t)\right)$ a.e. such that

$$
\xi(u)=\int_{0}^{1}\langle\bar{\eta}, u\rangle d s \quad \text { for any } u \in L^{1} .
$$

(e) Finally, for every $\xi \in \partial K_{2} \int_{0}^{1}\left|\dot{x}_{0}(t)-\Phi\left(t, x_{0}(t), u_{0}(t)\right)\right| d t$, there exists

$$
\left(\bar{p}, \mu_{1}, \mu_{2}\right) \in \partial_{(\dot{x}, x, u)} K_{2}\left|\dot{x}_{0}(t)-\Phi\left(t, x_{0}(t), u_{0}(t)\right)\right|
$$

such that

$$
\xi(x, u)=\int_{0}^{1}\left\{(\bar{p}, \dot{x}\rangle+\left\langle\mu_{1}, x\right\rangle+\left\langle\mu_{2}, u\right\rangle\right\} d t \quad \text { for any }(x, u) \in A C \times L^{1}
$$

Since $\partial_{(\dot{x}, x, u)}(\dot{x}-\Phi(x, u))=\left\{\left(1,-\Phi_{x,}-\Phi_{u}\right)\right\}$, by Jacobian Chain Rule, (5.10) implies that

$$
\left(\mu_{1}, \mu_{2}\right)=-\nabla_{(x, u)}\left\langle\bar{p}, \Phi\left(x_{0}, u_{0}\right)\right\rangle .
$$

Then, we arrive at 
LemMa 5.4. There exists $\bar{\lambda}_{i}, \xi_{i}, \eta_{i}, \nu_{i}(i=0 \cdots k), \bar{\eta}_{i}(i=1 \cdots k), \bar{\eta}, \theta_{1}, \theta_{2}, r, \bar{p}$, $\mu_{1}, \mu_{2}$ stated in the above, such that for any $(x, u) \in A C \times L^{1}$

$$
\begin{aligned}
0= & \sum_{i=0}^{k} \bar{\lambda}_{i} \int_{0}^{1}\left\{\left\langle\xi_{i}, x\right\rangle+\left\langle\eta_{i}, u\right\rangle\right\} d t+\sum_{i=0}^{k} \bar{\lambda}_{i}\left\langle\nu_{i}, x(1)\right\rangle+\sum_{i=1}^{k} \bar{\lambda}_{i} \int_{0}^{1}\left\langle\bar{\eta}_{i}, u\right\rangle d t \\
& +\left\langle\theta_{1}, x(0)\right\rangle+\int_{0}^{1}\left\langle\theta_{2}, u\right\rangle d t+\langle r, x\langle 0)\rangle+\int_{0}^{1}\langle\bar{\eta}, u\rangle d t \\
& \int_{0}^{1}\left\{(\bar{p}, \dot{x}\rangle+\left\langle\mu_{1}, x\right\rangle+\left\langle\mu_{2}, u\right\rangle\right\} d t .
\end{aligned}
$$

Separating $x$ and $u$, from the above equation we get

$$
\begin{gathered}
\int_{0}^{i}\left\langle\sum_{i=0}^{k} \bar{\lambda}_{i} \xi_{i}+\mu_{1}, x\right\rangle d t+\int_{0}^{1}\langle\bar{p}, \dot{x}\rangle d t+\left\langle\sum_{i=0}^{k} \bar{\lambda}_{i} \nu_{i}, x(1)\right\rangle+\left\langle\theta_{1}+r, x(0)\right\rangle=0 \\
\int_{0}^{1}\left\langle\sum_{i=0}^{k} \bar{\lambda}_{i} \eta_{i}+\mu_{2}+\sum_{i=1}^{k} \bar{\lambda}_{i} \bar{\eta}_{i}+\bar{\eta}+\theta_{2}, u\right\rangle d s=0 \quad \text { for } \text { any } u \in L^{1}
\end{gathered}
$$

Hence, (5.13) shows that

$$
\sum_{i=0}^{k} \bar{\lambda}_{i} \eta_{i}(t)+\mu_{2}(t)=-\sum_{i=1}^{k} \bar{\lambda}_{i} \bar{\eta}_{i}(t)-\bar{\eta}(t)-\theta_{2} \quad \text { a.e. }
$$

According to Dubois-Reymond Lemma (see Hestenes (1980)), using standard variational arguments, from (5.12), we observe that

$$
\begin{aligned}
& \bar{p}(t)=\int_{0}^{t}\left(\sum_{i=0}^{k} \bar{\lambda}_{i} \xi_{i}+\mu_{1}\right) d s+r+\theta_{1} \\
& \bar{p}(1)=-\sum_{i=0}^{k} \bar{\lambda}_{i} \nu_{i} \in-\sum_{i=0}^{k} \bar{\lambda}_{i} \partial h_{i}\left(x_{0}(1)\right) \\
& \bar{p}(0)=r+\theta_{1} \in K_{1} \partial d_{C}\left(x_{0}(0)\right)+\theta_{1} .
\end{aligned}
$$

By $(5.15)$, we see that $\dot{\bar{p}}(t)-\sum_{i=0}^{k} \bar{\lambda}_{i} \xi_{i}=\mu_{1}$. Letting $\bar{\zeta}=-\sum_{i=0}^{k} \bar{\lambda}_{i} \eta_{i}-\mu_{2}$, from (5.11), we have

$$
(-\bar{p}(t), \bar{\zeta}) \in \nabla_{(x, u)}\left\langle\bar{p}, \Phi\left(x_{0}, u_{0}\right)\right)-\sum_{i=1}^{k} \bar{\lambda}_{i} \partial_{(x, u)} L_{i}\left(t, x_{0}, u_{0}\right) \text { a.e. }
$$

Letting $\bar{\zeta}_{1}=\sum_{i=1}^{k} \bar{\lambda}_{i} \bar{\eta}_{i}+\theta_{2}$ and $\bar{\zeta}_{2}=\bar{\eta}$, by $(5.14),(\mathrm{a})$ and (d), we get

$$
\bar{\zeta}=\bar{\zeta}_{1}+\bar{\zeta}_{2}, \quad \bar{\zeta}_{1} \in-\sum_{i=1}^{k} \lambda_{i} \partial \Lambda_{i}\left(u_{0}(\cdot)\right)+\theta_{2}, \quad \bar{\zeta}_{2}(t) \in N_{U(t)}\left(u_{0}(t)\right) \text { a.e.. }
$$

Notice that $\bar{p}, \bar{\zeta}, \bar{\lambda}_{i}, i=0, \cdots, k$ depend on $\epsilon$. From (5.1), we know that $\left(x_{0}, u_{0}\right) \rightarrow$ $\left(x_{*}, u_{*}\right)$ as $\epsilon \rightarrow 0$. Letting $\epsilon \rightarrow 0$, as in the proof of Theorem 3.2.6 in Clarke (1983), 
from (5.16)-(5.19), it follows that there are $p \in A C, \zeta \in L^{\times}$and $\lambda_{i} \geq 0 . i=0, \cdots, k$ with $\sum_{t=0}^{k} \lambda_{1}=1$ such that $(2.1)-(2.3)$ hold.

Observe that for sufficient small $\epsilon>0$, if $i_{0} \in\left\{i \in[1, \cdots, k]: \bar{G}_{3}\left(x_{*}, u_{m}\right)<0\right\}$, then $\bar{G}_{i_{0}}\left(x_{0}, u_{0}\right)<0$. It is easy to see that $i_{0} \notin I_{0}\left(x_{0}, u_{0}\right)$. Hence, we have $\lambda_{i_{1}}=0$, which yields (2.4).

Finally, let (2.5) and (2.6) hold. If $\lambda_{0}=0$, then $\sum_{i=1}^{k} \lambda_{i}=1,(2.1)$ and (2.3) imply that

$$
\left(\dot{p}+p \Phi_{x}[t],-\zeta+p \Phi_{u}[t]\right) \in \sum_{i=1}^{k} \lambda_{i} \partial_{(x, u)} L_{i}[t], \quad-p(1) \in \sum_{i=1}^{k} \lambda_{i} \partial h_{i}\left(x_{*}(1)\right) .
$$

Then, from (2.2), (2.4)-(2.6) and (5.20), it follows that

$$
\begin{aligned}
0 & >\sum_{i=1}^{k} \lambda_{i}\left(\int_{0}^{1} L_{i}{ }^{\circ}\left(\left(x_{*}, u_{*}\right),\left(x_{1}, u_{1}\right)\right)(t) d t+h_{i}{ }^{\circ}\left(x_{*}, x_{1}\right)+\left(-\Lambda_{i}\right)_{i}^{o}\left(u_{*}, u_{1}\right)\right) \\
& \geq \int_{0}^{1}\left(\dot{p} x_{1}+p \Phi_{x}[t] x_{1}-\zeta u_{1}+p \Phi_{u}[t] u_{1}\right) d t-p(1) x_{1}(1)+\int_{0}^{1} \zeta_{1} u_{1} d t \\
& =\int_{0}^{1}\left(-p \dot{x}_{1}+p \Phi_{x}[t] x_{1}+p \Phi_{u}[t] u_{1}\right) d t-p(0) x_{1}(0)-\int_{0}^{1} \zeta_{2} u_{1} d t \\
& =-p(0) x_{1}(0)-\int_{0}^{1} \zeta_{2} u_{1} d t \\
& \geq 0
\end{aligned}
$$

which is a contradiction. Thus, we have $\lambda_{0} \neq 0$, which completes the proof.

Now, we give a simple example.

EXAMPLE 5.5. Let us consider the following trilevel dynamic optimization problem.

$$
\begin{aligned}
P_{1}: \quad \text { Minimize: } & x(1)+\int_{0}^{1}(|x-u|-v-w) d t \\
\text { subject to: } & \dot{x}=u-v-w \text { a.e., } x(0)=0 \\
& u \geq 0 \text { a.e. } \\
& v \in \mathcal{V}[u], w \in \mathcal{W}[u, v],
\end{aligned}
$$

where $\mathcal{V}[u]$ is the set of optimal controls $v$ of the following problem:

$$
\begin{aligned}
P_{2}[u]: \quad \text { Minimize }: & y(1)+\int_{0}^{1} y d t \\
\text { subject to }: & y=u+v \text { a.e., } y(0)=0 \\
& v \geq 0 \text { a.e., }
\end{aligned}
$$

$\mathcal{W}[u, v]$ is the set of optimal controls $w$ of the following problem,

$$
\begin{aligned}
P_{2}[u, v]: \text { Minimize : } & z(1)+\int_{0}^{1} z d t \\
\text { subject to }: & \dot{z}=u+v+w \text { a.e., } z(0)=0 \\
& w \geq 0 \text { a.e.. }
\end{aligned}
$$


Here, $(x, y, z) \in A C\left(\{0,1], R^{3}\right)$ is the state, $(u, v, w) \in L^{1}\left([0,1], R^{3}\right)$ is the control.

For this problem, it is easy to see that

$$
\begin{aligned}
F_{1}(u) & :=\inf \left\{y(1)+\int_{0}^{1} y d t: \dot{y}=u+v, y(0)=0, v \geq 0\right\} \\
& =\int_{0}^{1}\left(u+\int_{0}^{t} u d \tau\right) d t \\
V_{2}(u, v): & =\inf \left\{z(1)+\int_{0}^{1} z d t: \dot{z}=u+v+w, z(0)=0, w \geq 0\right\} \\
& =\int_{0}^{1}\left(u+v+\int_{0}^{t}(u+v) d \tau\right) d t
\end{aligned}
$$

Then, $P_{1}$ is equivalent to the following optimal control problem,

$$
\begin{aligned}
P_{1}: \quad \text { Minimize: } \quad & x(1)+\int_{0}^{1}(|x-u|-v-w) d t \\
\text { subject to: } \quad & \dot{x}=u-v-w \text { a.e., } x(0)=0 \\
& y=u+v \text { a.e., } y(0)=0 \\
& \dot{z}=u+v+w \text { a.e., } z(0)=0 \\
& u \geq 0, v \geq 0, w \geq 0 \text { a.e. } \\
& y(1)+\int_{0}^{1}\left(y-u-\int_{0}^{t} u d \tau\right) d t \leq 0 \\
& z(1)+\int_{0}^{1}\left(z-u-v-\int_{0}^{t}(u+v) d \tau\right) d t \leq 0 .
\end{aligned}
$$

If $\left(x_{*}, y_{*}, z_{*} ; u_{*}, v_{*}, w_{*}\right)$ is a local minimizer for $P_{1}$, then $v_{*}=w_{*}=0$ and by Theorem 2.1 there are $p_{1}, p_{2}, p_{3} \in A C, \zeta_{1}, \zeta_{2}, \zeta_{3} \in L^{\infty}$ and $\lambda_{1}, \lambda_{2}, \lambda_{3} \geq 0$ with $\sum_{i=1}^{3} \lambda_{i}=1$ such that (2.1)-(2.3) hold.

Here, (2.1) is equivalent to that

$$
\begin{aligned}
&\left(-\dot{p}_{1}, \zeta_{1}\right)=\left(0, p_{1}+p_{2}+p_{3}\right)-\lambda_{1} \alpha(1,-1)-\lambda_{2}\left(0, \partial_{u}\left(-u-\int_{0}^{t} u d \tau\right)\right) \\
&-\lambda_{3}\left(0, \partial_{v}\left(-v-\int_{0}^{t} v d \tau\right)\right) \text { a.e. } \\
& \quad \text { or some } \alpha \in[-1,1] \\
&-\dot{p}_{2}(t)=-\lambda_{2}, \zeta_{2}=-p_{1}+p_{2}+p_{3}+\lambda_{1}+\lambda_{3} \partial_{v}\left(v+\int_{0}^{t} v d \tau\right) \\
&-\dot{p}_{3}(t)=-\lambda_{2}, \zeta_{3}=-p_{1}+p_{3}+\lambda_{1} .
\end{aligned}
$$

(2.3) implies that

$$
\left.-p_{1}(1)=\lambda_{1},-p_{2}(1)=\lambda_{2},-p_{3}(1)\right)=\lambda_{3} .
$$

Combining (5.21) and (5.22) we see that

$$
p_{1}=\alpha \lambda_{1} t-\alpha \lambda_{1}-\lambda_{1}, \quad p_{2}=\lambda_{2} t-2 \lambda_{2}, \quad p_{3}=\lambda_{3} t-2 \lambda_{3},
$$

and for any $u_{0} \in L^{1}$

$$
\left\langle\zeta_{1}, u_{0}\right\rangle=\left(p_{1}+p_{2}+p_{3}\right) u_{0}+\left(\alpha \lambda_{1}+\lambda_{2}+\lambda_{3}\right) u_{0}+\left(\lambda_{2}+\lambda_{3}\right) \int_{0}^{t} u_{0} d \tau \text { a.e. }
$$


If $u_{*} \neq 0$, i.e. there exists $I \in[0,1]$ whose measure is not zero such that $u_{*}(t)>0$ for any $t \in I$, then from (2.2) it follows that $\zeta_{1}(t)=0$ for a.e. $t \in I$. Put $u_{0} \equiv 1,(5.24)$ implies that

$$
\alpha \lambda_{1} t-\lambda_{1}+2 \lambda_{2} t-\lambda_{2}+2 \lambda_{3} t-\lambda_{3}=0 \text { for a.e. } t \in I,
$$

which contradicts that $\lambda_{1}, \lambda_{2}, \lambda_{3} \geq 0$ and $\sum_{i=1}^{3} \lambda_{i}=1$. Therefore $u_{*}=0$.

Here, it is not difficult to check that there exist $\lambda_{1}>0$ with $p_{1}, p_{2}, p_{3}, \zeta_{1}, \zeta_{2}, \zeta_{3}$ and $\lambda_{2}, \lambda_{3}$ stated above such that (2.1) and (2.4) hold. Thus, by Theorem 2.2 we know that $\left(x_{*}, y_{*}, z_{*} ; u_{*}, v_{*}, w_{*}\right)=0$ is an optimal solution for $P_{1}$.

\section{Acknowledgements}

The author is greatly indebted to Professor K. Tsujioka for many helpful advices and constant encouragement. He would like to express his deep gratitude to Professor S. Koike for helpful suggestions. He also would like to thank the referee very much for useful comments.

\section{References}

Clarke, F. H. (1983). Optimization and Nonsmooth Analysis, Wiley-Intersicence, New York.

Clarke, F. H. (1975). Generalized gradients and applications, Trans. Amer. Math. Soc. 205,247-262.

Hestenes, M. R. (1980). Calculus of Variations and Optimal Control Theory, John Wiley,New York.

Loewen, P. D. (1993). Optimal Control via Nonsmooth Analysis, CRM Proceedings Lecture Notes 0.2, American Mathematical Society, Providence.

Pinho, M. R. and Vinter, R. B. (1995). An Euler-Lagrange inclusion for optimal Control Problems,IEEE Trans. Automat. control, 40, No.7,July.

Ye, J. J. (1995).Necessary conditions for bilevel dynamic optimization problems, SIAM $J$, Control Optim., 33,1208-1233.

Ye, J. J. (1997).Optimal strategies for bilevel dynamic problems, SIAM J, Control Optim., 35,512-531.

Ye, J. J. and Zhu, Q. J. (1997).Perturbed differential inclusion problems with nonaddictive $\mathrm{L}^{1}$ - perturbations and applications,J. Optim. Theory Appl., 92, No. 1,189-208.

Yezza, A. (1996).First-order necessary optimality conditions for general bilevel programming problems,J. Optim. Theory Appl.,89, No. 1, 189-219.

Received June 23,1998

Revised October 29,1998

Re-revised January 12,1999

Re-re-revised March 1,1999 